
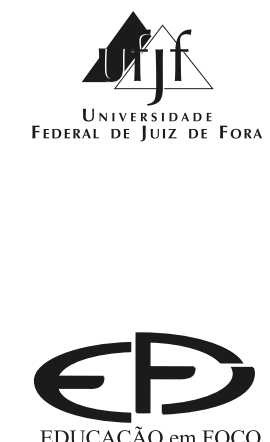

EDUCAÇÃO em FOCO

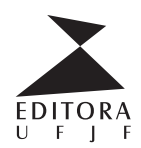

Faculdade de Educação

ograma de Pós-Graduaçãa em Educação
Reitor: Marcus Vinicius David

Vice-reitora: Girlene Alves da Silva

Diretor da Editora: Dmitri Cerboncini Fernandes

Diretor da Faculdade de Educação: Prof. Dr. André Silva Martins

Endereço para correspondência:

Faculdade de Educação / Centro Pedagógico

Campus Universitário da UFJF

Juiz de Fora -MG - CEP 36036-330

Telefone/Fax: (32) 2102-3653 / 2102-3656

E-mail: revista.edufoco@uff.edu.br

Home Page: https://educacaoemfoco.uff.emnuvens.com.br/edufoco/index

\section{Editora UFJF}

Rua Benjamin Constant, 790

Centro - Juiz de Fora - MG - CEP 36015-400

Telefone/Fax: (32) 3229-7646 / 3229-7645

MAMM (Museu de Arte Moderna Murilo Mendes)

E-mails: editora@editorauff.com.br / distribuicao.editora@uff.edu.br

Home Page: www.editorauff.com.br

\section{Ficha Técnica}

\begin{tabular}{|c|c|}
\hline Foto capa & Revisão Geral \\
\hline "Amarelinha" - Gervane de Paula & Paula Trivella \\
\hline Direito de reprodução & Indexadores \\
\hline $\begin{array}{l}\text { gentilmente cedido pelo autor. } \\
\text { Título: Amarelinha }\end{array}$ & - http://www.geodados.uem.br \\
\hline Técnica: óleo sobre tela & - BBE - Bibliografia Brasileira de Educação \\
\hline Dimensões: $160 \times 100 \mathrm{~cm}$ & - Sumários.org \\
\hline Contatos: Magna Domingos - & - DOAI - Directory of Open Acess Journals \\
\hline Comunicação e Gestão Cultural & - EZ3 - Elektronische Zeitschriftenbibliothek \\
\hline Dom Produções Ltda & - Portal de Periódicos da UFJF \\
\hline 55 (65) 9235-4295 / 2127-0373 & - www.latindex.unam.mx \\
\hline magnadom@gmail.com & - Portal de Periódicos CAPES \\
\hline Arte da capa e diagramaçấo & - Periódicos de Livre \\
\hline Breno Morozowski sob a & - Diadorim \\
\hline supervisão da Gráfica e Editora & - Ibict ser \\
\hline Copiart & - Google Acadêmico \\
\hline
\end{tabular}

Educação em Foco : revista de educação / Universidade Federal de Juiz de Fora, Faculdade de Educação, Centro Pedagógico - vol. 21, n. 3 (set. 2016/dez. 2016) - Juiz de Fora : EDUFJF, 2015-

Quadrimestral

Disponível em: http://educacaoemfoco.ufjf.emnuvens. com.br/edufoco

ISSN 0104-3293 (Versão impressa)

ISSN 2447-5246 (Versão on-line)

1. Educação - Periódicos. I. Universidade Federal de Juiz de Fora. Faculdade de Educação. Centro Pedagógico.

\section{CDU 37}

Ficha catalográfica elaborada por Adriana A. Oliveira - Bibliotecária - CRB6/1537

Nenhuma parte desta publicaçáo pode ser reproduzida por qualquer meio sem a prévia autorizaçáo da editora. 


\section{EDUCAÇÃO EM FOCO}

\section{EDITOR-CHeFE}

Prof. Dr. Aimberê Guilherme Quintiliano Rocha Amaral

Prof. ${ }^{a}$ Dr. ${ }^{a}$ Cristhiane Carneiro Flôr

\section{Conselho Editorial Executivo}

Prof. ${ }^{a}$ Dr. ${ }^{a}$ Adriana Rocha Bruno

Prof. Dr. Aimberê Guilherme Quintiliano Rocha Amaral

Prof. Dr. Alexandre José Pinto Cadilhe de Assis Jácome

Prof. ${ }^{a}$ Dr. ${ }^{a}$ Cristhiane Carneiro Flôr

Prof. Dr. Daniel Cavalcanti de Albuquerque Lemos

Prof. Dr. Jader Janer Moreira Lopes

Prof. ${ }^{a}$ Dr. ${ }^{a}$ Katiuscia Vargas Antunes

Prof. Dr. Wilson Alviano Júnior

Bibliotecária: Adriana A. Oliveira

Técnico Administrativo: Israel Pinheiro Marques

Bolsista de Treinamento Profissional: Tainara da Silva Campos

\section{Conselho Científico Internacional}

Prof. Dr. Abdeljalil Akkari - Universidade de Genebra - Suíça

Prof. Dr. Adrian Ascolani - Universidade Nacional de Rosário - Argentina

Prof. ${ }^{a}$ Dr. ${ }^{a}$ Ana Cecilia Vergara Del Solar - Universidade Diego Portales - Chile

Prof. Dr. Antônio Gomes Ferreira - Universidade de Coimbra - Portugal

Prof. Dr. Bernard Fichtner - Universidade de Siegen - Alemanha

Prof. Dr. Gilles Monceau - Université de Cergy-Pontoise - França

Prof. Dr. Guenadi Kravtsov - Universidade Russa de Humanidades - Rússia

Prof. ${ }^{a}$ Dr. ${ }^{a}$ Elena Kravtsova - Universidade Russa de Humanidades - Rússia

Prof. Dr. Fernando Bárcena - Universidade Complutense de Madrid - Espanha

Prof. Dr. Fernando Hernandez - Universidade Barcelona - Espanha

Prof. Dr. Hubert Vincent - Université de Rouen - França

Prof. a Irina Periliguina - Universidade Russa de Humanidadees - Rússia

Prof. Dr. Jean Hébrard - École des Hautes Études en Sciences Sociales - França

Prof. ${ }^{a}$ Dr. ${ }^{a}$ Julie Delalande - Université Caén - França

Prof. Dr. Leonid Pergamenschik - Universidade Estatal de Pedagogia da

Bielorússia - Bielorússia

Prof. Dr. Manuel Sarmento - Universidade do Minho - Portugal

Prof. a Dr. ${ }^{\text {a }}$ Maribel Vergara Arboleda - Universidad de San Buenaventura Colômbia

Prof. Dr. Michalis Kontopodis - Universidade de Roehampton - Inglaterra

Prof. ${ }^{a}$ Dr. ${ }^{a}$ Margarida Louro Felgueiras - Universidade do Porto - Portugal

Prof. ${ }^{a}$ Dr. ${ }^{a}$ Patricia Eliana Castillo Gallardo - Universidade Diego Portales Chile

Prof.a Dra. Patricia Medina Melgarejo - Universidad Nacional Autónoma de México UNAM - México

Prof. Dr. Serguei Jerebtsov - Universidade de Psicologia Francisk Skorina de Gomel - Bielorússia

Prof. Dr. Vladimir Sobkin - Instituto Federal de Educação da Academia de Ciências da Educação da Rússia - Rússia 


\section{Conselho Científico Nacional}

Prof. ${ }^{a}$ Dra Ana Icenicki - Universidade Federal do Rio de Janeiro

Prof. Dr a Ana Chrystina Venancio Mignot - Universidade do Estado do Rio de Janeiro

Prof. Dr. Amarilio Ferreira Junior - Universidade Federal de São Carlos

Prof. ${ }^{a}$ Dr. ${ }^{a}$ Carlos Henrique Carvalho - Universidade Federal de Uberlândia

Prof. ${ }^{a}$ Dr. ${ }^{a}$ Clarice Nunes - Universidade Federal Fluminense

Prof. Dr. Cleiton de Oliveira - Universidade Metodista de Piracicaba

Prof. ${ }^{a}$ Dr. ${ }^{a}$ Daniela Freire - Universidade Federal do Mato Grosso

Prof. ${ }^{a}$ Dr. ${ }^{a}$ Diana Gonçalves Vidal - Universidade de São Paulo

Prof. ${ }^{a}$ Dr. ${ }^{a}$ Edméia Oliveira dos Santos - Universidade do Estado do Rio de Janeiro

Prof. ${ }^{a}$ Dr. ${ }^{a}$ Flávia Motta - Universidade Federal Rural do Rio de Janeiro

Prof. Dr. Irlen Antônio Gonçalves - Centro Federal Tecnológico de Minas Gerais

Prof. Dr. José Silvério Baia Horta - Universidade Federal do Amazonas

Prof. Dr. Laerthe de Moraes Abreu Junior - Universidade Federal de São João

Del Rey

Prof. ${ }^{a}$ Dr. ${ }^{a}$ Lia Ciomar Macedo Faria - Universidade do Estado do Rio de Janeiro

Prof. Dr. Luciano Mendes de Faria Filho - Universidade Federal de Minas Gerais

Prof. ${ }^{a}$ Dr. ${ }^{a}$ Magda Becker Soares - Universidade Federal de Minas Gerais

Prof. ${ }^{a}$ Dr. ${ }^{a}$ Magda Damiani - Universidade Federal de Pelotas

Prof. Dr. Marcelo Soares Pereira da Silva - Universidade Federal de Uberlândia

Prof. Dr. Marcio da Costa - Universidade Federal do Rio de Janeiro

Prof. ${ }^{a}$ Dr. ${ }^{a}$ Maria Letícia Nascimento - Universidade de São Paulo

Prof. ${ }^{a}$ Dr. ${ }^{a}$ Maria de Lurdes de A. Fávero - Universidade Federal do Rio de Janeiro

Prof. a Dr. ${ }^{a}$ Maria Teresa Assunção Freitas - Universidade Federal de Juiz de Fora

Prof. ${ }^{a}$ Dr. ${ }^{a}$ Maria Teresa Eglér Mantoan - Universidade Estadual de Campinas

Prof. ${ }^{a}$ Dr. ${ }^{a}$ Maria Vilanir Cosme de Carvalho - Universidade Federal do Piauí

Prof. ${ }^{a}$ Dr. ${ }^{a}$ Marisa Bittar - Universidade Federal de São Carlos

Prof. ${ }^{a}$ Dr. ${ }^{a}$ Marisol Barenco de Mello - Universidade Federal Fluminense

Prof. ${ }^{a}$ Dr. ${ }^{a}$ Neuza Salim - Universidade Federal de Juiz de Fora

Prof. a Dr. ${ }^{a}$ Nilda Alves - Universidade do Estado do Rio de Janeiro

Prof. Dr. Osmar Fávero - Universidade Federal Fluminense

Prof. ${ }^{a}$ Dr. ${ }^{a}$ Rosemary Dore Heijmans - Universidade Federal de Minas Gerais

Prof. a Dr. ${ }^{a}$ Rosimar de Fátima Oliveira - Universidade Federal de Minas Gerais

Prof. Dr. Rubem Barbosa Filho - Universidade Federal de Juiz de Fora

Prof. ${ }^{a}$ Dr. ${ }^{a}$ Sandra Zákia - Universidade de São Paulo

Prof. ${ }^{\text {a }}$ Dr. ${ }^{\text {a }}$ Sonia de Castro Lopes - Universidade Federal do Rio de Janeiro

Prof. ${ }^{a}$ Dr. ${ }^{a}$ Suzani Cassiani - Universidade Federal de Santa Catarina

Prof. ${ }^{a}$ Dr. ${ }^{a}$ Terezinha Oliveira - Universidade Estadual de Maringá

Prof. Dr. Tiago Adão Lara - Universidade Federal de Uberlândia

Prof. Dr. Ubiratan D. Ambrósio - Universidade Estadual de Campinas

Prof. Dr. Wenceslau Gonçalves Neto - Universidade Federal de Uberlândia

Prof. ${ }^{a}$ Dr. ${ }^{a}$ Zoia Prestes - Universidade Federal Fluminense 